\title{
Comparison between biparametric and multiparametric MR imaging of Prostate Imaging Reporting and Data System Version 2.1 in detection of prostate cancer
}

Mohamed Ali EL-Adalany', Ahmed Abd E L-khalek Abd EL-Razek', Tarek EL-Diasty², Ahmed EL-Hendy² and Dina EL-Metwally ${ }^{1 *}$

\begin{abstract}
Background: Prostate cancer (PCa) is considered to be the commonest cancer among males. Early and precise diagnosis of $\mathrm{PCa}$ is essential for adequate treatment. Multiparametric MR imaging (mpMRI) is actually the most precise imaging technique used for early diagnosis of PCa. The aim of this work was to assess the diagnostic capability of biparametric MRI (bpMRI) and multiparametric MRI (mpMRI) of PI-RADS V2.1 in detection of prostate cancer (PCa). This prospective study was carried on 60 male patients with high PSA. bpMRI and mpMRI were performed for all patients using a 3-T MRI scanner. The diagnostic performance of bpMRI of PI-RADS V2.1 was compared to that of mpMRI of PI-RADS V 2.1. The diagnosis of Pca was confirmed by transrectal ultrasound-guided biopsy and the results of open prostatectomy specimens.

Results: When considering PI-RADS categories 1, 2, and 3 as benign and categories 4 and 5 as malignant, mpMRI had higher sensitivity and diagnostic accuracy when compared with bPMRI (sensitivity was $88.6 \%$ for mpMRI versus $60 \%$ for bpMRI and diagnostic accuracy was $91.7 \%$ for mpMRI versus $75 \%$ for bpMRI). When considering PI-RADS categories 1 and 2 as benign and PI-RADS categories 3.4 and 5 as malignant, the sensitivity and diagnostic accuracy of bpMRI and mpMRI were comparable (sensitivity was $94.3 \%$ for both bpMRI and mpMRI and diagnostic accuracy was $86.7 \%$ for both bpMRI and mpMRI).

Conclusion: Considering PI-RADS scores 4 and 5 as malignant, mpMRI had higher sensitivity and diagnostic accuracy when compared with bpMRI; however, when considering PI-RADS scores 3, 4, and 5 as malignant, both bpMRI and mpMRI had similar diagnostic accuracy.
\end{abstract}

Keywords: Prostate, Cancer, bpMRI, mpMRI, DWI, T2WI, DCE

\section{Background}

Prostate cancer ( $\mathrm{PCa}$ ) is considered to be the commonest cancer among males and is considered as the second major cause of mortality induced by cancer in males in the USA. Early and precise diagnosis of $\mathrm{PCa}$ is essential for adequate treatment. Several modalities are available

\footnotetext{
* Correspondence: elmetwallydina@gmail.com

${ }^{1}$ Faculty of Medicine, Mansoura University, Mansoura, Egypt

Full list of author information is available at the end of the article
}

for examination of males suspected to have cancer prostate as serum prostate-specific antigen (PSA) levels, digital rectal examination, and transrectal ultrasound (TRUS). However, all these modalities have limited sensitivity and specificity in the early diagnosis of $\mathrm{PCa}[1$, 2]. Multiparametric MR imaging (mpMRI) is actually the most precise imaging technique used for early diagnosis of PCa [3]. It helps in local staging and assessment of the aggressiveness of $\mathrm{PCa}$ [4]. According to prostate imaging reporting and data system (PI-RADS) version 2

\section{Springer Open}

() The Author(s). 2021 Open Access This article is licensed under a Creative Commons Attribution 4.0 International License, which permits use, sharing, adaptation, distribution and reproduction in any medium or format, as long as you give appropriate credit to the original author(s) and the source, provide a link to the Creative Commons licence, and indicate if changes were made. The images or other third party material in this article are included in the article's Creative Commons licence, unless indicated otherwise in a credit line to the material. If material is not included in the article's Creative Commons licence and your intended use is not permitted by statutory regulation or exceeds the permitted use, you will need to obtain permission directly from the copyright holder. To view a copy of this licence, visit http://creativecommons.org/licenses/by/4.0/. 
and version 2.1, mpMRI consists of T2-weighted imaging (T2WI), diffusion-weighted imaging (DWI), and dynamic contrast enhancement (DCE) [5]. Like PIRADS V 2, In PI-RADS 2.1, DCE has a minor role in the detection of clinically significant prostate cancer (csPca) (DCE is fundamentally utilized to raise lesions in the peripheral zone (PZ) from category 3 to category 4 based on positive findings on DCE). The only difference between PI-RADS V 2 and PI-RADS V 2.1 is that positive and negative enhancement on DCE was better illustrated on PI-RADS V 2.1 [6].

There is usually debate regarding the role of DCE in the diagnosis of $\mathrm{PCa}$. Some previous studies stated that the diagnostic performance of biparametric MRI (bpMRI) (T2WI and DWI) is nearly similar to that of mpMRI in the detection of csPca, and they recommended omitting DCE-MRI from the protocol used for the diagnosis of Pca because it is time-consuming and also because of the side effects of contrast media [7-12]. On the other hand, other studies reported that adding DCE to DWI improved the accuracy of Pca detection especially for tumors located in the peripheral zone [4, 13]. Despite the controversy about the role of DCE, the PI-RADS steering committee still recommended its inclusion in the mpMRI protocol in the PI-RADS V 2.1 and they recommended that further multiple readers and multicenter studies are required to evaluate the role of bPMRI and evaluate the additive value of DCE. Only a few recent studies discussed the value of PI-RADS v2.1 in the assessment of $\mathrm{PCa}$ [14-16]. The unique of this study is that we compared bpMRI with mpMRI of PIRADS v2.1 in the diagnosis of PCa at 3-T MRI.

The aim of this work is to assess the diagnostic capability of bPMRI and mpMRI of PI-RADS V2.1 in the detection of PCa.

\section{Methods}

\section{Patient's demographic data}

Approval from our institution's ethics committee was acquired, and informed consents were acquired from all patients before inclusion in this work. This prospective study included 60 male patients with clinically suspected prostate cancer; their age ranged from 48 to 82 years, and the mean age \pm SD was $65 \pm 8$ years. Patients did not receive chemotherapy, radiotherapy, or hormonal therapy prior to the MRI examinations. No biopsies were performed to all patients prior to the MRI examinations.

\section{Inclusion criteria}

Patients with clinically suspected prostate cancer by digital rectal examination or by high serum PSA ( $>4 \mathrm{ng} /$ dl) were included.

\section{Exclusion criteria}

Patients who had contraindications to do MRI as patients with cardiac pace maker, with cochlear implant, and ocular foreign body; patients who refused contrast media injection; patients who had contraindications for contrast media injection as patients with impaired renal function; patients with poor quality of the MR images as patients with motion artifact; and patients who were lost and their pathological results were not available were excluded.

\section{MRI technique}

The procedure was conducted using a 3-T MRI scanner (Ingenia, Philips medical systems, Veenpluis, Netherlands). Patients lied in the supine position, and an abdominal eightchannel surface phased array coil was well fitted on the pelvis. The region of interest was taken from the urinary bladder to the end of the prostate. The following sequences were obtained for all patients: (1) T2-weighted images (field of view (FOV): $200 \mathrm{~mm}$, repetition time (TR): $5000 \mathrm{msec}$, echo time (TE): $110 \mathrm{msec}$, slice thickness: $3 \mathrm{~mm}$, no interslice gap, and matrix: $288 \times 192$ ). According to the recommendations of the PI-RADS version 2.1, T2W images were acquired in axial (straight axial), sagittal, and coronal planes. (2) Diffusionweighted images (DWI) (FOV: $350 \mathrm{~mm}$, TR: $7255 \mathrm{msec}$, TE: $85 \mathrm{msec}$, slice thickness: $3 \mathrm{~mm}$, no interslice gap and matrix: $128 \times 96)$. Regarding the $b$ values, we used low $\left(0 \mathrm{~s} / \mathrm{mm}^{2}\right)$, intermediate $\left(800 \mathrm{~s} / \mathrm{mm}^{2}\right)$, and high $\left(1400 \mathrm{~s} / \mathrm{mm}^{2}\right) b$ values. ADC maps were obtained from DW images at b0 and b1400 $\mathrm{s} / \mathrm{mm}^{2}$ gradients. (3) Dynamic contrast enhancement (DCE) (FOV: $200 \mathrm{~mm}$, TR: $19 \mathrm{msec}$, TE: $1.93 \mathrm{msec}$, slice thickness: $4 \mathrm{~mm}$, no interslice gap, temporal resolution $<15 \mathrm{~s}$ and matrix: $320 \times 192)$. DCE images were obtained after IV injection of contrast media (Dotarem (Gadoteric acid)) at a dose of $0.1 \mathrm{mmol} / \mathrm{kg}$ (maximum dose $15 \mathrm{mmol}$ ) at a rate of 3 $\mathrm{mL} / \mathrm{s}$. A contrast injection was performed using an automatic injector.

\section{Image interpretation}

Biparametric and multiparametric MR images were analyzed on the basis of the PI-RADS version 2.1 by 2 radiologists (EA, ME) with 5 and 14 years' experience in uroradiology. The two radiologists analyzed the biparametric MR images at first (T2WI, DWI, and ADC), and then, subsequently on the same setting, the multiparametric MR images were analyzed. The two radiologists were blinded to the results of histopathological examination. On the basis of the PI-RADS version 2.1 all detected lesions were assigned a score from 1 to 5 representing the probability of clinically significant $\mathrm{PCa}$ (PI-RADS 1: very low, PI-RADS 2: low, PI-RADS 3: intermediate, PI-RADS 4: high, PI-RADS 5: very high). For the transitional zone (TZ) lesions, the scoring was done mainly on T2WI. Category 2 lesions on T2WI 

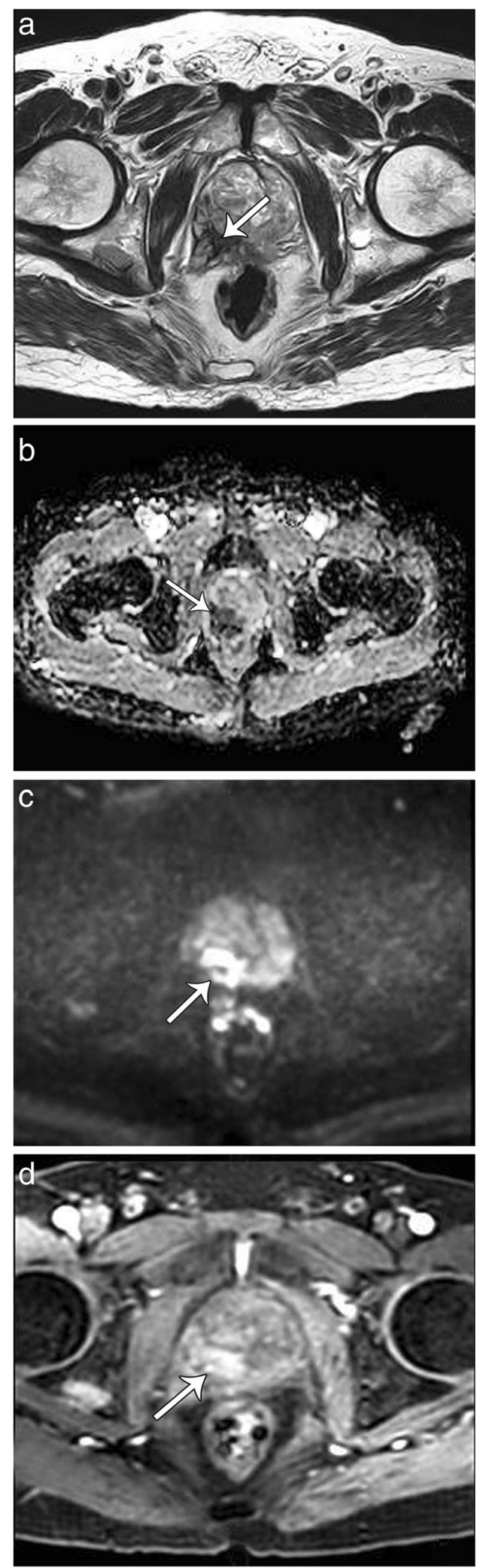

Fig. 1 Prostatic adenocarcinoma with Gleason score $3+4$ in a 65year-old male patient with serum PSA $>100 \mathrm{ng} / \mathrm{ml}$. a Axial T2Wl shows circumscribed homogeneous moderate hypointense mass $>$ $1.5 \mathrm{~cm}$ seen involving the right posterolateral peripheral zone of the mid prostate. $\mathbf{b}$ Axial ADC shows focal markedly hypointense mass > $1.5 \mathrm{~cm}$. c Axial high $b$ value DWI shows focal markedly hyperintense mass $>1.5 \mathrm{~cm}$ (the final PI-RADS score on basis bpMRI was score 5). d Axial DCE shows +ve contrast enhancement (the final PI-RADS score on the basis of mpMRI was score 5, DCE did not aid in the diagnosis of this case and it was already diagnosed by bpMRI)

were raised to category 3 when the lesions were categorized as 4 or 5 on DWI. Category 3 lesions on T2WI were raised to category 4 when they were categorized as 5 on DWI. For peripheral zone (PZ) lesions, the scoring was done mainly on DWI. Category 3 lesions on DWI were raised to category 4 when they showed positive enhancement on DCE on mpMRI. On bpMRI, the categorization of PZ lesion was done only on the basis of DWI and the lesions on category 3 will remain in category 3 and not upgraded. In cases with multifocal lesions, we reported only the index lesion for statistical analysis. On basis of PI-RADS version2.1, the index lesion is defined as the lesion that shows the highest PIRADS assessment category, and if the highest PI-RADS Assessment Category is specified to more than or equal two lesions, the index lesion is the lesion that revealed extraprostatic extension (EPE). If EPE was not present in any of the detected lesions, the index lesion will be the lesion that showed the largest dimensions and the highest PI-RADS Assessment Category. The two radiologists joined and reached a correspondence about the bpMRI and mpMRI scores for controversial cases.

\section{Final diagnosis}

TRUS-guided biopsy (standard 12-core random systematic biopsy) was performed for all patients after the MRI studies. The duration between the MRI study and the histopathological examination was 7-10 days. TRUSguided biopsy was done by using (Flex focus 500, bk medical, Herlev, Denmark) with high-frequency transrectal transducer $(5-9 \mathrm{MHz})$ with a condom cover. The patient lied in a lithotomy position. A lubricant gel is used with lignocaine cream before insertion of the probe. Axial and coronal scans were obtained to demonstrate the volume of the prostate, any abnormal foci at the peripheral zone, and the infiltration of the seminal vesicles by malignancies. Twelve cores were taken, and tissue biopsies were sent for histopathologic evaluation.

When TRUS-guided biopsy revealed PCa, open prostatectomy was performed and the diagnosis of prostatic carcinoma was confirmed. According to PI-RADS v2.1, csPCa is diagnosed by histopathology as the lesion with Gleason score more than 7 (including $3+4$ with 

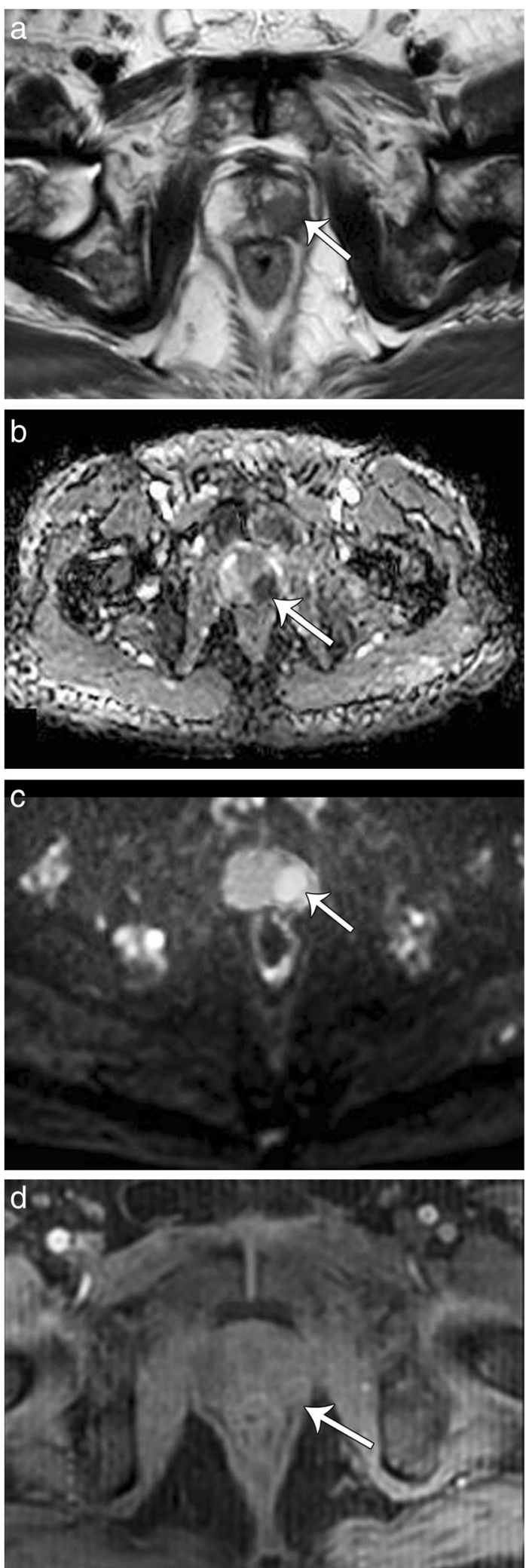

Fig. 2 Prostatic adenocarcinoma with Gleason score $4+4$ in a 71-year-old male patient with serum PSA $=70 \mathrm{ng} / \mathrm{ml}$. a Axial T2WI shows circumscribed homogeneous moderate hypointense mass $>1.5 \mathrm{~cm}$ seen involving the left posterolateral and left posteromedial peripheral zones of the mid prostate. b Axial ADC shows focal markedly hypointense mass $>1.5 \mathrm{~cm}$. $\mathbf{c}$ Axial high $b$ value DWI shows focal markedly hyperintense mass $>1.5 \mathrm{~cm}$ (the final PI-RADS score on basis bpMRI was score 5). $\mathbf{d}$ Axial DCE shows -ve contrast enhancement (the final PI-RADS score on the basis of mpMRI was score 5, DCE did not aid in the diagnosis of this case and it was already diagnosed by bpMRl)

prominent but not predominant Gleason 4 component), and/or volume more than $0.5 \mathrm{cc}$, and/or EPE. In order to match the MRI findings with the results of the prostatectomy specimen, we reported the site of the index lesion on MRI according to the sector map proposed by the PI-RADS version 2.1, and subsequently, we obtained the prostatectomy results for the related zones.

\section{Statistical analysis and data interpretation}

Data were fed to the computer and analyzed using IBM SPSS Corp. (released in 2013, IBM SPSS Statistics for Windows, Version 22.0. Armonk, NY: IBM Corp). Qualitative data were described using numbers and percentages. The significance of the obtained results was judged at the (0.05) level. Diagnostic accuracy for categorical variables was detected by cross-tabulation to detect true positive and true negative, and then a calculation of sensitivity, specificity, positive predictive rate, negative predictive rate, and accuracy. The sensitivity, specificity, positive predictive value (PPV), negative predictive value (NPV), and diagnostic accuracy of both bpMRI and mpMRI of PI-RADS version 2.1 were calculated two times, one time when considering PI-RADS categories 4 and 5 as malignant lesions and the other time when considering PI-RADS categories 3,4 , and 5 as malignant lesions.

\section{Results}

This study included 60 male patients with clinical suspicion of prostate cancer. The PSA level for patients included in this study ranged from 4.8 to $100 \mathrm{ng} / \mathrm{dl}$ (mean $\pm \mathrm{SD}=35 \pm 33.9 \mathrm{ng} / \mathrm{dl}$ ), and the mean PSA level for malignant cases was mean $\pm \mathrm{SD}=50.3 \pm 33.9 \mathrm{ng} / \mathrm{dl}$. According to the histopathology, 35 patients $(58.3 \%)$ were malignant (adenocarcinoma) and 25 patients were benign (41.7\%). Among those with benign lesions, 22 patients were $\mathrm{BPH}(88 \%)$ and 3 patients were prostatitis (12\%). Out of the pathologically proven 25 benign lesions, 22 lesions were located at the $\mathrm{TZ}$ and 3 lesions were located at the PZ. Out of the pathologically proven 35 malignant lesions, 31 lesions were located at the PZ and 4 lesions were located at the TZ. Out of the $31 \mathrm{ma}$ lignant lesions located at the PZ, 7 lesions were located in the right mid prostate, 6 lesions were located in the 
Table 1 Score and number of malignant and benign prostatic lesions on pbMRI and mpMRI

\begin{tabular}{|c|c|c|c|c|c|c|}
\hline \multirow[t]{2}{*}{ Pathology } & \multicolumn{3}{|l|}{ bpMRI } & \multicolumn{3}{|l|}{ mpMRI } \\
\hline & $\begin{array}{l}\text { Score } \\
1-2(N=21)\end{array}$ & $\begin{array}{l}\text { Score } 3 \\
(N=17)\end{array}$ & $\begin{array}{l}\text { Scores 4-5 } \\
(N=22)\end{array}$ & $\begin{array}{l}\text { Scores 1-2 } \\
(N=21)\end{array}$ & $\begin{array}{l}\text { Score } 3 \\
(N=7)\end{array}$ & $\begin{array}{l}\text { Scores 4-5 } \\
(N=32)\end{array}$ \\
\hline Benign & 19 & 5 & 1 & 19 & 5 & 1 \\
\hline Malignant & 2 & 12 & 21 & 2 & 2 & 31 \\
\hline
\end{tabular}

left mid prostate, 5 lesions were located in the right prostate apex, 3 lesions were located in the left prostate apex, 4 lesions were located in the right prostate base, and 6 lesions were located in the left prostate base. After open prostatectomy, the Gleason score for the 35 malignant cases were $3+3$ for 2 patients (5.7\%), $3+4$ for 7 patients (20\%), $4+3$ for 9 patients (25.7\%), $4+4$ for 16 patients (45.8\%), and $4+5$ for 1 patient (2.8\%).

When comparing the final PI-RADS assessment categories of bpMRI and mpMRI with the results of histopathological examination, we found that the total PIRADS v2.1 score of bpMRI revealed 22 lesions with scores 4 and 5 (Figs. 1 and 2), 21 out of them were proved to be malignant and only one lesion was proved to be benign. Twenty-one lesions had scores 1 and 2, 2 lesions out of them were proved to be malignant and 19 lesions were proved to be benign. Indeterminate lesions (score 3) were 17 lesions, 12 out of them were proved to be malignant and 5 lesions were proved to be benign. The total PI-RADs v2.1 score of mpMRI revealed 32 lesions with scores 4 and 5 (Figs. 1 and 2), 31 lesions out of them were proved to be malignant and only one lesion was benign, and 21 lesions with scores 1 and 2, 19 lesions out of them were benign and 2 lesions were malignant. Indeterminate lesions (score 3 ) were 7 lesions, 2 out of them were proved to be malignant and the remaining 5 lesions were proved to be benign (Table 1).

When considering PI-RADS scores 4 and 5 only as malignant lesions, we found that mpMRI had higher sensitivity and diagnostic accuracy when compared with bpMRI. mpMRI had $88.6 \%$ sensitivity, 96\% specificity, 96.9\% PPV, 86.7\% NPV, and 91.7\% diagnostic accuracy, while bpMRI had 60\% sensitivity, 96\% specificity, 95.5\% PPV, 63.2\% NPV, and $75 \%$ diagnostic accuracy (Table 2). When considering PI-RADS scores 3, 4, and 5 as malignant lesions, we found that the sensitivity and diagnostic accuracy of both bpMRI and mpMRI were comparable. Both bpMRI and mpMRI had $94.3 \%$ sensitivity, $76 \%$ specificity, $84.6 \%$ PPV, $90.5 \%$ NPV, and 86.7\% diagnostic accuracy (Table 3).

\section{Discussion}

The main finding in this research work is that mpMRI had higher sensitivity and diagnostic accuracy when compared with bpMRI considering PI-RADS categories 4 and 5 only as malignant lesions, while when considering PI-RADS categories 3, 4, and 5 as malignant lesions, the sensitivity and diagnostic accuracy of both mpMRI and bpMRI were comparable.

PI-RADS V2.1 is an updated new scoring system for $\mathrm{PCa}$ detection, preserving the frame of assigning scores to single sequences and then employing these scores to obtain a gross assessment category. Several previous studies investigated the additive value of DCE to T2WI and DWI in PCa detection on the basis of PI-RADS v. 2, and there was controversy regarding the role of DCE. Several studies found that the sensitivity and diagnostic accuracy of bPMRI (T2WI and DWI) were comparable to that of mpMRI (T2WI, DWI, and DCE) [7, 8, 11, 17-20]. It was stated that the performance of bpMRI and mpMRI in the diagnosis of PCa was nearly similar (AUC for bpMRI = 0.914 and AUC for mpMRI = 0.917) [17]. One study performed a meta-analysis of the head-to-head comparison between the diagnostic accuracy of bpMRI and mpMRI and concluded that the sensitivity and specificity of bPMRI and mpMRI were nearly similar ( $74 \%$ sensitivity and $90 \%$ specificity for bpMRI versus $76 \%$ sensitivity and

Table 2 Diagnostic values for bpMRI and mpMRI in detection of prostate cancer when considering PI-RADS scores 1, 2, and 3 as benign and PI-RADS scores 4 and 5 as malignant

\begin{tabular}{|c|c|c|c|c|c|c|c|}
\hline & \multicolumn{2}{|c|}{ Pathology } & \multirow{2}{*}{$\begin{array}{l}\text { Sensitivity } \\
\text { (\%) }\end{array}$} & \multirow{2}{*}{$\begin{array}{l}\text { Specificity } \\
\text { (\%) }\end{array}$} & \multirow{2}{*}{$\begin{array}{l}\text { PPV } \\
\text { (\%) }\end{array}$} & \multirow{2}{*}{$\begin{array}{l}\text { NPV } \\
\text { (\%) }\end{array}$} & \multirow{2}{*}{$\begin{array}{l}\text { Accuracy } \\
\text { (\%) }\end{array}$} \\
\hline & $\overline{\text { BG }}$ & MG & & & & & \\
\hline \multicolumn{8}{|l|}{ bpMRI } \\
\hline Benign (PI-RADS 1, 2, and 3) & 24 & 14 & 60.0 & 96.0 & 95.5 & 63.2 & 75.0 \\
\hline Malignant (PI-RADS 4 and 5) & 1 & 21 & & & & & \\
\hline \multicolumn{8}{|l|}{ mpMRI } \\
\hline Benign (PI-RADS 1, 2, and 3) & 24 & 4 & 88.6 & 96.0 & 96.9 & 85.7 & 91.7 \\
\hline Malignant (PI-RADS 4 and 5) & 1 & 31 & & & & & \\
\hline
\end{tabular}


Table 3 Diagnostic values for bpMRI and mpMRI in detection of prostate cancer when considering PI-RADS scores 1 and 2 as benign and PI-RADS scores 3, 4, and 5 as malignant

\begin{tabular}{|c|c|c|c|c|c|c|c|}
\hline & \multicolumn{2}{|c|}{ Pathology } & \multirow{2}{*}{$\begin{array}{l}\text { Sensitivity } \\
\text { (\%) }\end{array}$} & \multirow{2}{*}{$\begin{array}{l}\text { Specificity } \\
\text { (\%) }\end{array}$} & \multirow{2}{*}{$\begin{array}{l}\text { PPV } \\
\text { (\%) }\end{array}$} & \multirow{2}{*}{$\begin{array}{l}\text { NPV } \\
\text { (\%) }\end{array}$} & \multirow{2}{*}{$\begin{array}{l}\text { Accuracy } \\
\text { (\%) }\end{array}$} \\
\hline & $\overline{\text { BG }}$ & MG & & & & & \\
\hline \multicolumn{8}{|l|}{ bpMRI } \\
\hline Benign (PI-RADS 1 and 2) & 19 & 2 & 94.3 & 76.0 & 84.6 & 90.5 & 86.7 \\
\hline Malignant (PI-RADS 3, 4, and 5) & 6 & 33 & & & & & \\
\hline \multicolumn{8}{|l|}{ mpMRI } \\
\hline Benign (PI-RADS 1 and 2) & 19 & 2 & 94.3 & 76.0 & 84.6 & 90.5 & 86.7 \\
\hline Malignant (PI-RADS 3, 4, and 5) & 6 & 33 & & & & & \\
\hline
\end{tabular}

$89 \%$ specificity for mpMRI) [7]. Another study stated that the diagnostic performance of both bpMRI and mpMRI was similar with AUC of 0.91 for bpMRI and 0.93 for mpMRI [8]. It was stated that the diagnostic accuracy of both bpMRI and mpMRI was comparable $(89.1 \%$ accuracy of bpMRI versus $87.2 \%$ accuracy for mpMRI) [11]. A previous study concluded that the difference between the diagnostic accuracy of bpMRI and mpMRI was not statistically significant (83\% accuracy for bpMRI versus $82 \%$ accuracy for mpMRI for reader 1 and $80 \%$ accuracy for bpMRI versus $82 \%$ accuracy for mpMRI for reader 2 ) [18]. It was reported that both bpMRI and mpMRI had comparable diagnostic accuracy in PCa diagnosis [19]. One study stated that in men with suspicious lesions on mpMRI (PI-RADS scores 3-5), both bpMRI and mpMRI had similar sensitivity (95\%) and bpMRI and mpMRI had specificity of $65 \%$ and $69 \%$, respectively [20]. To our knowledge, this study is a unique study that compares the diagnostic performance of bPMRI and mpMRI on the basis of the PI-RADS V 2.1, and we found that the sensitivity, specificity, and diagnostic accuracy of bpMRI and mpMRI were comparable when considering PI-RADS scores 3,4 , and 5 as malignant lesions.

On the other hand, in PI-RADS v 2.1, the PI-RADS steering committee still recommend the inclusion of DCE to the protocol of mpMRI used for diagnosis of prostate cancer and stated that DCE is considered as a secure sequence, particularly when T2WI or DWI showed poor image quality due to artifacts or inappropriate signal-tonoise ratio (SNR), a condition which is common to occur on some MRI devices when prostate MRI was carried out without using an endorectal coil. It was stated that DCE could increase tumor detection rate by about $16 \%$ for score 3 PZ lesions on DWI [13]. Also, it was stated that for lesions with category more than or equal 3 on bpMRI, DCE was found to be of statistical significance in the detection of PCa [4]. One study concluded that readers' experience is mandatory for assessment of bpMRI where the sensitivity of bPMRI and mpMRI were $91 \%$ and $96 \%$, respectively, for expert readers, while for less-experienced readers mpMRI had higher sensitivity when compared with bpMRI (sensitivity was 58\% for bpMRI versus $91 \%$ for mpMRI) [21]. In this work, mpMRI showed higher sensitivity and diagnostic accuracy when compared with bPMRI when considering malignant lesions as lesions with PI-RADS scores 4 and 5 only.

There are a few limitations of this study: The first one was a small number of studied patients. Further research works with a larger number of patients and multicenter studies are recommended. Second, the body surface coil was used in examination and not an endorectal coil. Third, it would be better to perform this study with multiple readers and assess the interobserver agreement.

\section{Conclusion}

Considering PI-RADS scores 4 and 5 as malignant, mpMRI had higher sensitivity and diagnostic accuracy when compared with bpMRI; however, when considering PI-RADS scores 3, 4, and 5 as malignant, both bPMRI and mpMRI had similar diagnostic accuracy.

\section{Abbreviations}

bpMRI: Biparametric magnetic resonance imaging; DWI: Diffusion-weighted imaging; DCE: Dynamic contrast enhancement; mpMRI: Multiparametric magnetic resonance imaging; PI-RADS V2.1: Prostate Imaging Reporting and Data System (version 2.1); PCa: Prostate cancer; csPCa: Clinically significant prostate cancer; PSA: Prostatic specific antigen; TRUS: Transrectal ultrasound; PZ: Peripheral zone; TZ: Transitional zone; TR: Repetition time; TE: Echo time; FOV: Field of view; EPE: Extraprostatic extension; PPV: Positive predictive value; NPV: Negative predictive value

\section{Acknowledgements}

Not applicable.

\section{Authors' contributions}

AA and TE revised the collected data and the manuscript. AE and ME analyzed the MRI images of all patients. DM wrote the manuscript and performed the statistical analysis. The authors read and approved the final manuscript.

\section{Funding}

No funding resources.

Availability of data and materials

The datasets used and/or analyzed during the current study are available from the corresponding author on reasonable request. 


\section{Ethics approval and consent to participate}

The study was approved by our institution's ethics committee (Mansoura Faculty of Medicine Institutional Research Board) (ethics committee reference number is MS/17.06.30), and all patients gave their written informed consent before inclusion in the study.

\section{Consent for publication}

All patients included in this research gave written informed consent to publish the data contained within this study. If the patient was less than 16 years old, deceased, or unconscious when consent for publication was requested, written informed consent for the publication of this data was given by their parent or legal guardian.

\section{Competing interests}

The authors declare no competing interests.

\section{Author details}

${ }^{1}$ Faculty of Medicine, Mansoura University, Mansoura, Egypt. ${ }^{2}$ Urology \& Nephrology Center, Mansoura University, Mansoura, Egypt.

Received: 12 November 2020 Accepted: 14 February 2021

Published online: 02 March 2021

\section{References}

1. Cornud F (2020) Bi-parametric prostate MRI before biopsy: yes, but only if you deserve it. Diagn Interv Imaging 101:191-192. https://doi.org/10.1016/j. diii.2020.03.001

2. Han C, Liu S, Qin XB, Ma S, Zhu LN, Wang XY (2020) MRI combined with PSA density in detecting clinically significant prostate cancer in patients with PSA serum levels of 4 10ng/mL: biparametric versus multiparametric MRI. Diagn Interv Imaging 101:235-244. https://doi.org/10.1016/j.diii.2020.01.014

3. Turkbey B, Rosenkrantz AB, Haider MA, Padhani AR, Villeirs G, Macura K et al (2019) Prostate Imaging Reporting and Data System Version 2.1: 2019 update of Prostate Imaging Reporting and Data System Version 2. Eur Urol 76(3):340-351. https://doi.org/10.1016/j.eururo.2019.02.033

4. Xu L, Zhang G, Shi B, Liu Y, Zou T, Yan W et al (2019) Comparison of biparametric and multiparametric MRI in the diagnosis of prostate cancer. Cancer Imaging 19:90. https://doi.org/10.1186/s40644-019-0274-9

5. Weinreb JC, Barentsz JO, Choyke PL, Cornud F, Haider M, Macura KJ et al (2016) PI-RADS Prostate Imaging - Reporting and Data System: 2015, version 2. Eur Urol 69:16-40. https://doi.org/10.1016/j.eururo.2015.08.052

6. Ullrich T, Schimmöller $L$ (2020) Perspective: a critical assessment of PI-RADS 2.1. Abd Radiol. https://doi.org/10.1007/s00261-020-02424-7

7. Woo S, Suh CH, Kim SY, Cho JY, Kim SH, Moon MH (2018) Head-to-head comparison between biparametric and multiparametric MRI for the diagnosis of prostate cancer: a systematic review and meta-analysis. Am J Roentgenol 211:226-241. https://doi.org/10.2214/AJR.18.19880

8. Stanzione A, Imbriaco M, Cocozza S, Fusco F, Rusconi G, Nappi C et al (2016) Biparametric 3T magnetic resonance imaging for prostatic cancer detection in a biopsy-naive patient population: a further improvement of PI-RADS v2? Eur J Radiol 85:2269-2274. https://doi.org/10.1016/j.ejrad.2016.10.009

9. Hansford BG, Peng Y, Jiang Y, Vannier MW, Antic T, Thomas S et al (2015) Dynamic contrast-enhanced MR imaging curve-type analysis: is it helpful in the differentiation of prostate cancer from healthy peripheral zone? Radiology. 275:448-457. https://doi.org/10.1148/radiol.14140847

10. Scialpi M, Prosperi E, D'Andrea A, Martorana E, Malaspina C, Palumbo B et al (2017) Biparametric versus multiparametric MRI with non-endorectal coil at $3 \mathrm{~T}$ in the detection and localization of prostate cancer. Anticancer Res 37: 1263-1271. https://doi.org/10.21873/anticanres.11443

11. Kuhl CK, Bruhn R, Kramer N, Nebelung S, Heidenreich A, Schrading S (2017) Abbreviated biparametric prostate MR imaging in men with elevated prostate-specific antigen. Radiology 285:493-505. https://doi.org/10.1148/ra diol.2017170129

12. Di Campli E, Delli Pizzi A, Seccia B, Cianci R, D'Annibale M, Colasante A et al (2018) Diagnostic accuracy of biparametric vs multiparametric MRI in clinically significant prostate cancer: comparison between readers with different experience. Eur J Radiol:10117-10123. https://doi.org/10.1016/j.ejra d.2018.01.028

13. Greer MD, Shih JH, Lay N, Barrett T, Bittencourt LK, Borofsky S et al (2017) Validation of the dominant sequence paradigm and role of dynamic contrast-enhanced imaging in PI-RADS version 2. Radiology 285:859-869. https://doi.org/10.1148/radiol.2017161316

14. Tamada T, Kido A, Takeuchi M, Yamamoto A, Miyaji Y, Kanomata N et al (2019) Comparison of PI-RADS version 2 and PI-RADS version 2.1 for the detection of transition zone prostate cancer. Eur J Radiol 121:108704. https://doi.org/10.1016/j.ejrad.2019.108704

15. Brembilla G, Dell'Oglio P, Stabile A, Damascelli A, Brunetti L, Ravelli $S$ et al (2020) Interreader variability in prostate MRI reporting using Prostate Imaging Reporting and Data System version 2.1. Eur Radiol. https://doi.org/1 0.1007/s00330-019-06654-2

16. Byun J, Park KJ, Kim MH, Kim JK (2020) Direct comparison of PI-RADS Version 2 and 2.1 in transition zone lesions for detection of prostate cancer: preliminary experience. J Magn Reson Imaging. https://doi.org/10.1002/ jmri.27080

17. Junker D, Steinkohl F, Fritz V, Bektic J, Tokas T, Aigner F et al (2019) Comparison of multiparametric and biparametric MRI of the prostate: are gadolinium-based contrast agents needed for routine examinations? World J Urol 37:691-699. https://doi.org/10.1007/s00345-018-2428-y

18. Cho J, Ahn H, Hwang S, Lee HJ, Choe G, Byun S (2020) Biparametric versus multiparametric magnetic resonance imaging of the prostate: detection of clinically significant cancer in a perfect match group. Prostate Int. https:// doi.org/10.1016/j.prnil.2019.12.004

19. Kang Z, Min X, Weinreb J, Li Q, Feng Z, Wang L (2019) Abbreviated biparametric versus standard multiparametric MRI for diagnosis of prostate cancer: a systematic review and meta-analysis. AJR Am J Roentgenol:357365. https://doi.org/10.2214/AJR.18.20103

20. van der Leest M, Israël B, Cornel EB, Zámecnik P, Schoots IG, van der Lelij H et al (2019) High diagnostic performance of short magnetic resonance imaging protocols for prostate cancer detection in biopsy-naive men: the next step in magnetic resonance imaging accessibility. Eur Urol 76(5):574581. https://doi.org/10.1016/j.eururo.2019.05.029

21. Gatti M, Faletti R, Calleris G, Giglio J, Berzovini C, Gentile F (2019) etal., Prostate cancer detection with biparametric magnetic resonance imaging (bpMRI) by readers with different experience: performance and comparison with multiparametric (mpMRI). Abdom Radiol 44(5):1883-1893. https://doi. org/10.1007/s00261-019-01934-3

\section{Publisher's Note}

Springer Nature remains neutral with regard to jurisdictional claims in published maps and institutional affiliations.

\section{Submit your manuscript to a SpringerOpen ${ }^{\bullet}$ journal and benefit from:}

- Convenient online submission

- Rigorous peer review

- Open access: articles freely available online

- High visibility within the field

- Retaining the copyright to your article

Submit your next manuscript at $>$ springeropen.com 